\title{
CORDIC II: A New Improved CORDIC Algorithm
}

Mario Garrido Gálvez, Petter Källström, Martin Kumm and Oscar Gustafsson

\author{
Linköping University Post Print
}

\section{Tweet}

N.B.: When citing this work, cite the original article.

(C2016 IEEE. Personal use of this material is permitted. However, permission to reprint/republish this material for advertising or promotional purposes or for creating new collective works for resale or redistribution to servers or lists, or to reuse any copyrighted component of this work in other works must be obtained from the IEEE.

Mario Garrido Gálvez, Petter Källström, Martin Kumm and Oscar Gustafsson, CORDIC II: A New Improved CORDIC Algorithm, 2016, IEEE Transactions on Circuits and Systems - II Express Briefs, (63), 2, 186-190.

http://dx.doi.org/10.1109/TCSII.2015.2483422

Postprint available at: Linköping University Electronic Press

http://urn.kb.se/resolve?urn=urn:nbn:se:liu:diva-126139 


\title{
CORDIC II: A New Improved CORDIC Algorithm
}

\author{
Mario Garrido, Member, IEEE, Petter Källström, Martin Kumm and Oscar Gustafsson, Senior Member, IEEE
}

\begin{abstract}
In this paper we present the CORDIC II algorithm. Like previous CORDIC algorithms, the CORDIC II calculates rotations by breaking down the rotation angle into a series of micro-rotations. However, the CORDIC II algorithm uses a novel angle set, different from the angles used in previous CORDIC algorithms. The new angle set provides a faster convergence that reduces number of adders with respect to previous approaches.
\end{abstract}

Index Terms-CORDIC, rotation, friend angles, USR CORDIC, nano-rotation

\section{INTRODUCTION}

$\mathbf{T}$ HE CORDIC algorithm [1] is the algorithm par excellence to calculate rotations in digital systems. Its main principle is simple: It breaks down the rotation angle in a sum of angles, and carries out the rotation by a series of the so called micro-rotation by these angles. The benefit of the CORDIC algorithm is that the micro-rotations are calculated by simple shift-and-add operations, which is very efficient in hardware.

Many variations of the CORDIC algorithm have been proposed in the literature. In this paper we are interested in those approaches that are used to calculate general rotations. This means that they rotate by any angle provided as an input of the rotator. Constant rotators used for specific sets of rotation angles are not considered in this paper, but are studied in [2].

Among general rotators we find numerous versions of the CORDIC algorithm. Some works combine several microrotation stages into a single stage [3], [4] in order to reduce the number of iterations of the CORDIC. The work in [5] is based on skipping and/or repeating micro-rotations. Some approaches focus on representing the micro-rotation using a Taylor series approximation [6]-[8]. Other approaches divide the micro-rotations into a coarse and a fine part [9]. Some works focus on reducing the rotation memory [9]-[11]. Scaling-free CORDIC approaches pursue to compensate the scale factor of the CORDIC [6], [7]. Reviews of CORDIC techniques can be found in [12], [13].

In this paper we pursue a pipelined CORDIC design with the minimum number of adders. We call it the CORDIC II algorithm. It differs from previous approaches in the used set of micro-rotations, called angle set in the following. The new set of micro-rotations provides a fast convergence of the rotation angle. This leads to a reduced latency and a smaller number of adders than in previous CORDIC algorithms.

M. Garrido, P. Källström and O. Gustafsson are with the Division of Computer Engineering, Department of Electrical Engineering, Linköping University, SE-581 83 Linköping, Sweden, e-mails: \{mario.garrido.galvez, petter.kallstrom, oscar.gustafsson\}@liu.se

M. Kumm is with the Digital Technology Group, University of Kassel, 34121 Kassel, Germany, e-mail: kumm@uni-kassel.de

Copyright (c) 2015 IEEE. Personal use of this material is permitted. However, permission to use this material for any other purposes must be obtained from the IEEE by sending an email to pubs-permissions@ieee.org.

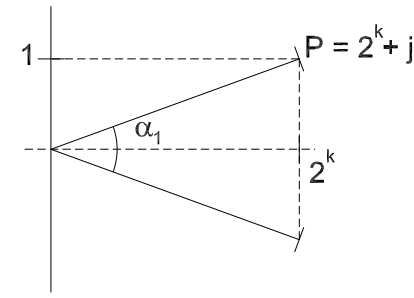

(a)

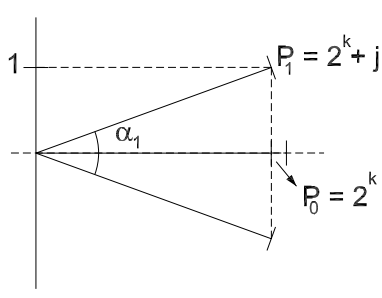

(b)
Fig. 1. CORDIC micro-rotation angles. (a) Conventional CORDIC. (b) Redundant CORDIC.

\section{BACKGROUND}

\section{A. Rotations in Digital Systems}

This section reviews key concepts related to rotations in digital systems. Further information can be found in [2], [14].

In a digital system, a rotation by an angle $\alpha$ can be described as a multiplication by a complex coefficient $P=C+j S$,

$$
\left[\begin{array}{c}
X_{D} \\
Y_{D}
\end{array}\right]=\left[\begin{array}{rr}
C & -S \\
S & C
\end{array}\right]\left[\begin{array}{l}
x \\
y
\end{array}\right]
$$

where $x+j y$ is the input and $X_{D}+j Y_{D}$ is the result of the rotation. $C$ and $S$ are $b$-bit integer numbers in 2's complement in the range $\left[-2^{b-1}, 2^{b-1}-1\right]$. They are obtained from the rotation angle as [14]

$$
\begin{aligned}
& C=R \cdot\left(\cos \alpha+\epsilon_{c}\right) \\
& S=R \cdot\left(\sin \alpha+\epsilon_{s}\right),
\end{aligned}
$$

where $\epsilon_{c}$ and $\epsilon_{s}$ are the quantization errors of the cosine and sine components, respectively, and $R$ is the scaling factor. The output $X_{D}+j Y_{D}$ is also scaled by $R$.

The rotation error $\epsilon=\sqrt{\epsilon_{c}^{2}+\epsilon_{s}^{2}}$ is the distance between the exact rotation and the actual rotation due to quantization. If the rotator has multiple rotation angles $\alpha_{i}, i=1, \ldots, M$, with their corresponding coefficients $P_{i}=C_{i}+j S_{i}$, the rotation error [14] is calculated as

$$
\epsilon=\max _{i}(\epsilon(i))=\max _{i}\left(\sqrt{\epsilon_{c}^{2}(i)+\epsilon_{s}^{2}(i)}\right) .
$$

Finally, the effective word length is the number of bits of the output that are guaranteed to be accurate [2] and is calculated from the rotation error as

$$
W L_{E}=-\log _{2} \frac{\epsilon}{2 \sqrt{2}}=-\log _{2} \epsilon+\frac{3}{2} .
$$

\section{B. The CORDIC Algorithm}

The CORDIC algorithm considers the coefficients $P=C+$ $j S=2^{k}+j \delta_{k}$, where $\delta_{k} \in\{-1,1\}$ and $k=0, \ldots, M$ is the micro-rotation stage. The corresponding angles are $\alpha_{k}=$ $\tan ^{-1}(S / C)=\delta_{k} \tan ^{-1}\left(2^{-k}\right)$. This is shown in Fig. 1(a). 


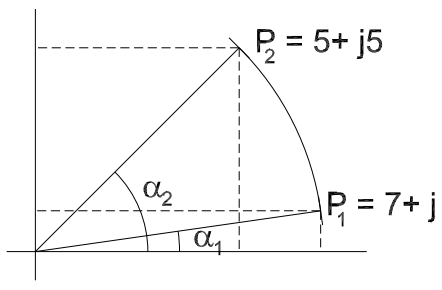

Fig. 2. Example of friend angles for $P_{1}=7+j$ and $P_{2}=5+j 5$.

The CORDIC algorithm breaks down the rotation angle $\theta$ into a sum of micro-rotations by the angles $\alpha_{k}$, i.e.,

$$
\theta=\sum_{k=0}^{M} \alpha_{k}+\epsilon_{\phi}
$$

where $\epsilon_{\phi}$ is the remaining phase error.

Each micro-rotation stage calculates

$$
\left[\begin{array}{c}
X_{D} \\
Y_{D}
\end{array}\right]=\left[\begin{array}{cc}
2^{k} & -\delta_{k} \\
\delta_{k} & 2^{k}
\end{array}\right]\left[\begin{array}{l}
x \\
y
\end{array}\right],
$$

where $\delta_{k}$ determines the direction of the rotation, and the scaling factor of the stage is $R(k)=\sqrt{2^{2 k}+1}$.

The rotation error at each micro-rotation stage is $\epsilon=0$ and the word length is $W L_{E}=\infty$. This means that the coefficient $P_{k}$ rotates exactly $\alpha_{k}$ degrees and the scaling factor for both angles in each micro-rotation is the same. The latter is always true, as the coefficients are conjugated.

\section{Redundant CORDIC Algorithm}

The redundant CORDIC [15] algorithm uses the same set of coefficients $P_{1}=C_{1}+j S_{1}=2^{k}+j \delta_{k}$ as the CORDIC algorithm, with the particularity that $\delta_{k} \in\{-1,0,1\}$. This adds a rotation $P_{0}=2^{k}$ by $0^{\circ}$ in the kernel, as shown in Fig. 1(b). This increases the set of alternative angles per stage, which implies a faster convergence to the rotation angle. However, it has the drawback that the scaling of the angles of the kernel is different. Therefore, redundant CORDIC algorithms need special stages to compensate the scaling and, thus, reduce the rotation error.

\section{BASIC ANGLE SETS}

There are three new types of angle sets proposed for CORDIC II, which are described in the following.

\section{A. Friend Angles}

We define friend angles as a set of angles $\alpha_{i}$ for which there exists a set of coefficients $P_{i}=C_{i}+j S_{i}$ with angles $\alpha_{i}$, i.e., $\alpha_{i}=\tan ^{-1}\left(S_{i} / C_{i}\right)$, whose magnitude is the same, i.e., $\forall i, j,\left|P_{i}\right|=\left|P_{j}\right|$. As all the coefficients have the same magnitude, a kernel composed by friend angles $\alpha_{i}$ does not have any rotation error. This is equivalent to say that $W L_{E}=$ $\infty$.

The angles $\alpha_{1}=8.13^{\circ}$ and $\alpha_{2}=45^{\circ}$ are an example of friend angles. For these angles there exist the coefficients $P_{1}=7+j$ and $P_{2}=5+j 5$ whose angles are $\alpha_{1}$ and $\alpha_{2}$, respectively, and $\left|P_{1}\right|=\left|P_{2}\right|=\sqrt{50}$. This example is shown in Fig. 2.

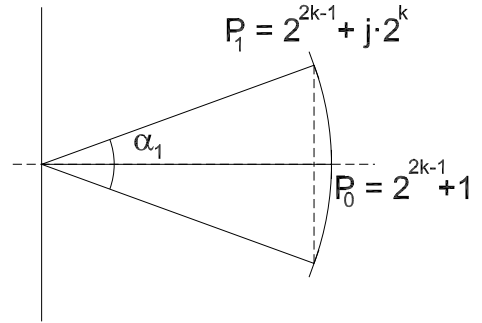

(a)

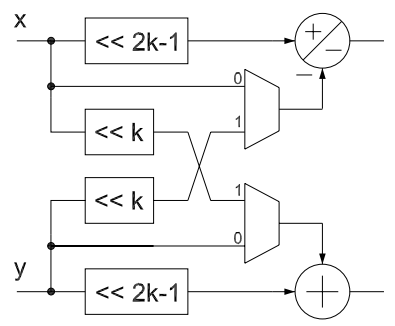

(b)
Fig. 3. USR CORDIC. (a) Graphical representation of the coefficients. (b) Hardware circuit.

TABLE I

UNIFORMLY-SCALED REDUNDANT CORDIC ROTATIONS.

\begin{tabular}{|c|cc|cc|c|c|}
\hline$k$ & $P_{0}$ & $\alpha_{0}$ & $P_{1}$ & $\alpha_{1}$ & $R$ & $W L_{E}$ \\
\hline \hline 1 & 3 & 0 & $2+\mathrm{j} 2$ & 45 & 2.91 & 6.59 \\
2 & 9 & 0 & $8+\mathrm{j} 4$ & 26.5651 & 8.97 & 9.83 \\
3 & 33 & 0 & $32+\mathrm{j} 8$ & 14.0362 & 32.99 & 13.59 \\
4 & 129 & 0 & $128+\mathrm{j} 16$ & 7.125 & 128.99 & 17.52 \\
5 & 513 & 0 & $512+\mathrm{j} 32$ & 3.5763 & 512.99 & 21.51 \\
6 & 2049 & 0 & $2048+\mathrm{j} 64$ & 1.7899 & 2048.99 & 25.50 \\
7 & 8193 & 0 & $8192+\mathrm{j} 128$ & 0.89517 & 8193.00 & 28.50 \\
8 & 32769 & 0 & $32768+\mathrm{j} 256$ & 0.44761 & 32769.00 & 32.50 \\
\hline
\end{tabular}

A property that can be extracted from the definition of friend angles is that any angle $\alpha$ is friend to itself and also to $-\alpha+n \pi / 2$ and $\alpha+n \pi / 2$ for any value of $n$. According to this property, the angles used in the CORDIC for each microrotation stage are friend angles. This happens because each micro-rotation only considers the pair of angles $\pm \alpha_{k}$.

\section{B. Uniformly-Scaled Redundant CORDIC}

The uniformly-scaled redundant (USR) CORDIC rotations use the same rotation angles as the redundant CORDIC. However, all the angles have similar scaling. The coefficients for the USR CORDIC are

$$
\begin{aligned}
& P_{0}=2^{2 k-1}+1 \\
& P_{1}=2^{2 k-1}+j 2^{k}
\end{aligned}
$$

and the graphical representation of the USR CORDIC is shown in Fig. 3(a). It can be observed that the magnitude of $P_{0}$ and $P_{1}$ is almost the same: From (7) we obtain $\left|P_{0}\right|^{2}=\left|P_{1}\right|^{2}+1$.

The angles of the USR CORDIC are

$$
\begin{aligned}
& \alpha_{0}=0 \\
& \alpha_{1}=\tan ^{-1}\left(\frac{2^{k}}{2^{2 k-1}}\right)=\tan ^{-1}\left(2^{-k+1}\right) .
\end{aligned}
$$

Table I shows a list of USR CORDIC rotators for different values of $k$. The table shows the coefficients $P_{0}$ and $P_{1}$ with their corresponding angles, the radius and $W L_{E}$, calculated as explained in [2], [14]. It can be observed that the first kernels have small $W L_{E}$. However, for large $k, W L_{E}$ is large enough to use them as rotation stages.

The hardware implementation of the USR CORDIC rotator is shown in Fig. 3(b). The figure shows that the USR CORDIC rotators are implemented using only 2 adders, like the conventional CORDIC rotations.

\section{Nano-Rotations}

Nano-rotations refer to the kernel formed by the coefficient set

$$
P_{k}=C+j k, \quad k=0, \ldots, N,
$$




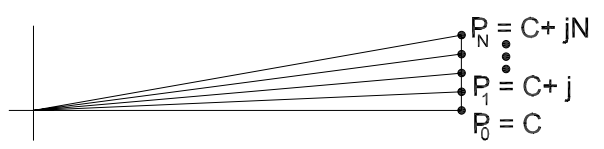

Fig. 4. Kernel to calculate nano-rotations.

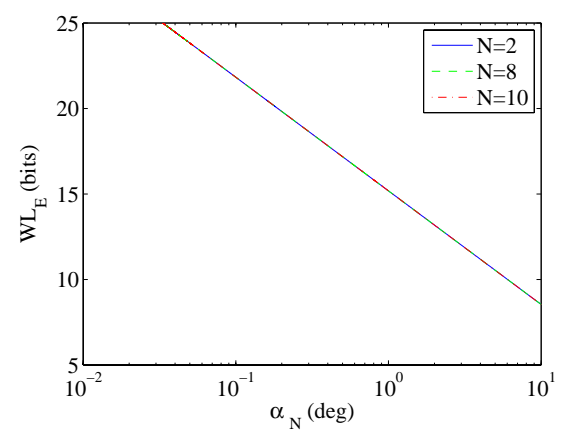

Fig. 5. Nano-rotations: $W L_{E}$ (bits) as a function of the angle $\alpha_{N}$ (deg).

where $C$ is constant and the corresponding angles are

$$
\alpha_{k}=\tan ^{-1}\left(\frac{k}{C}\right)
$$

In (9), $N$ is considered to be much smaller than $C$. This makes $\alpha_{k}$ small and fulfills $\alpha_{k} \approx \tan \left(\alpha_{k}\right)$. This leads to $\alpha_{k} \approx k / C$, which is a kernel with equally distributed angles. The fact that $N \ll C$ also makes the scaling of the coefficients very similar.

Figure 4 shows the kernel to calculate nano-rotations. It can be observed that the angle changes by simply changing the value of the imaginary part.

Figure 5 shows the $W L_{E}$ as a function of the largest angle of the kernel, $\alpha_{N}$, where

$$
\alpha_{N}(\mathrm{rad})=\tan ^{-1}\left(\frac{N}{C}\right) \approx \frac{N}{C}
$$

Note that $W L_{E}$ only depends on $\alpha_{N}$ independently of the number of angles, $N$. Figure 5 shows that $W L_{E}$ larger than 15 bits is achieved for angles smaller than $\alpha_{N}=1^{\circ}$. Thus, nano-rotations will be used when $\alpha_{N} \leq 1^{\circ}$.

To design the rotator, the angle $\alpha_{N}$ must be selected first, according to the range of input angles. Then, $N$ is selected. Finally, the constant $C$ is obtained from (11).

\section{Connecting Rotation Stages}

The CORDIC II algorithm consist of several rotation stages connected in series. Each rotation stage can be characterized by an input range $\left[-\alpha_{\text {in }}, \alpha_{\text {in }}\right]$, and an output range $\left[-\alpha_{\text {out }}, \alpha_{\text {out }}\right]$. For instance, the input angle for the CORDIC micro-rotation by $7.125^{\circ}$ is in the range $\left[-14.25^{\circ}, 14.25^{\circ}\right]$ and the output angle is in the range $\left[-7.125^{\circ}, 7.125^{\circ}\right]$.

In general, a rotation stage may include any number of rotation angles. Each input is rotated by one of these angles. We define an $N$-rotator as a rotator with $N$ different angles to choose from.

If $N$ is even, the rotator includes $N / 2$ coefficients and their conjugates. Figure 6(a) shows this case. The values of $\delta_{i}$ are defined as

$$
\begin{gathered}
\delta_{1}=\alpha_{1} \\
\delta_{i}=\left(\alpha_{i}-\alpha_{i-1}\right) / 2 \quad i=2, \ldots, N / 2
\end{gathered}
$$

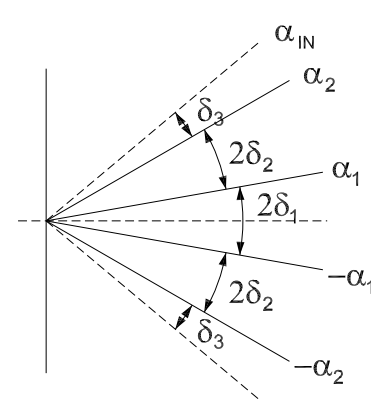

(a)

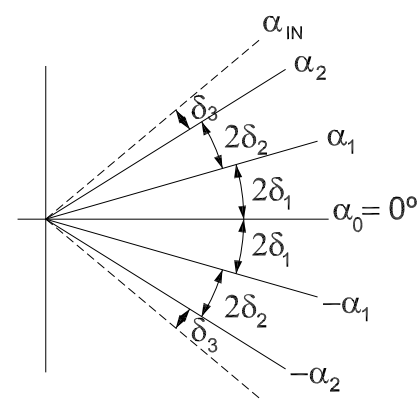

(b)
Fig. 6. Rotations of $N$-rotator. (a) $N$ even $(N=4)$. (b) $N$ odd $(N=5)$.

According to this, the input and output angles are

$$
\begin{gathered}
\alpha_{\text {out }}=\max _{i}\left(\delta_{i}\right) \quad i=1, \ldots, N / 2 \\
\delta_{N / 2+1}=\alpha_{\text {out }} \\
\alpha_{\text {in }}=\alpha_{N / 2}+\delta_{N / 2+1}=\alpha_{N / 2}+\alpha_{\text {out }}
\end{gathered}
$$

The best case happens when all the values of $\delta_{i}$ are equal, i.e., $\delta_{i}=\phi$, where $\phi$ is a constant. This minimizes the remaining angle to the next stage, $\alpha_{\text {out }}$. Under this conditions, $\alpha_{\text {out }}=$ $\phi=\alpha_{\text {in }} / N$, and the rotation angles are $\alpha_{i}=(2 i-1) \alpha_{\text {in }} / N$.

If $N$ is odd, the rotator includes $(N-1) / 2$ coefficients with their conjugates plus the coefficient for $\alpha=0^{\circ}$. Fig. 6(b) shows this case. The values of $\delta_{i}$ are defined as

$$
\delta_{i}=\left(\alpha_{i}-\alpha_{i-1}\right) / 2 \quad i=1, \ldots,(N-1) / 2
$$

According to this, the input and output angles are

$$
\begin{gathered}
\alpha_{\text {out }}=\max _{i}\left(\delta_{i}\right) \quad i=1, \ldots,(N-1) / 2 \\
\delta_{(N+1) / 2}=\alpha_{\text {out }} \\
\alpha_{\text {in }}=\alpha_{(N-1) / 2}+\delta_{(N+1) / 2}=\alpha_{(N-1) / 2}+\alpha_{\text {out }}
\end{gathered}
$$

The best case also happens when all the values of $\delta_{i}$ are equal, leading to $\alpha_{\text {out }}=\phi=\alpha_{\text {in }} / N$. In this case, the rotation angles are $\alpha_{i}=2 i \alpha_{\text {in }} / N$.

From this analysis we can draw several conclusions. First, in the best cases when the rotation angles are selected carefully, an $N$-rotator reduces the input range a factor $N$, because $\alpha_{\text {out }}=\phi=\alpha_{\text {in }} / N$. This fact justifies the efficiency of the CORDIC rotator, where many of the micro-rotations halve the rotation angle using a 2-rotator. Second, in order to design efficient rotators, we have to aim to rotators for which $\alpha_{\text {out }} \approx \phi=\alpha_{\text {in }} / N$. Finally, in order to connect stages in series, for each stage $\alpha_{\text {in }}$ must be larger than $\alpha_{\text {out }}$ of the previous stage. This guarantees the convergence of the rotation angle.

\section{The CORDIC II Algorithm}

Figure 7 shows the architecture of the CORDIC II rotator, and Table II includes detailed information about each rotation stage. The CORDIC II algorithm consists of six rotation stages in pipeline that use the angle sets describes in previous sections.

Stage 1: The first stage calculates trivial rotations by $\pm 180^{\circ}$ and $\pm 90^{\circ}$ to set the remaining angle in the range of $\pm 45^{\circ}$. The hardware architecture for the trivial rotator is shown in Fig. 8. 


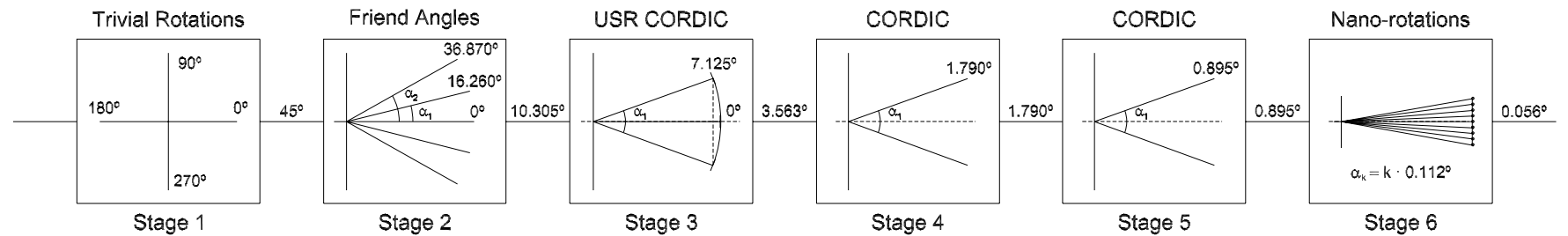

Fig. 7. Architecture of the CORDIC II rotator.

TABLE II

CORDIC II ROTATION STAGES.

\begin{tabular}{|c|c|c|c|c|c|c|c|c|c|}
\hline Stage & Rotator type & \multicolumn{2}{|c|}{ Micro-rotation } & \# Add. & \# Mux. & $W L_{E}$ & $\alpha_{\text {in }}$ & $\alpha_{\text {out }}$ & $R_{\text {norm }}$ \\
\hline 1 & $\begin{array}{l}\text { Trivial } \\
\text { rotations }\end{array}$ & $\begin{array}{l}P_{0}=1 \\
P_{1}=j \\
P_{2}=-1 \\
P_{3}=-j\end{array}$ & $\begin{array}{l}\alpha_{0}=0^{\circ} \\
\alpha_{1}=90^{\circ} \\
\alpha_{2}=180^{\circ} \\
\alpha_{3}=270^{\circ}\end{array}$ & 1 & 4 & $\infty$ & $\pm 180^{\circ}$ & $\pm 45^{\circ}$ & 1 \\
\hline 2 & Friend angles & $\begin{array}{l}P_{0}=25 \\
P_{1}=24+j 7 \\
P_{2}=20+j 15\end{array}$ & $\begin{array}{l}\alpha_{0}=0^{\circ} \\
\alpha_{1}=16.260^{\circ} \\
\alpha_{2}=36.870^{\circ}\end{array}$ & 5 & $7(+2)$ & $\infty$ & $\pm 47.175^{\circ}$ & $\pm 10.305^{\circ}$ & 1.563 \\
\hline 3 & $\begin{array}{c}\text { USR } \\
\text { CORDIC }\end{array}$ & $\begin{array}{l}P_{0}=129 \\
P_{1}=128+j 16\end{array}$ & $\begin{array}{l}\alpha_{0}=0^{\circ} \\
\alpha_{1}=7.125^{\circ}\end{array}$ & 2 & $2(+2)$ & 17.52 & $\pm 10.688^{\circ}$ & $\pm 3.563^{\circ}$ & 1.008 \\
\hline 4 & CORDIC & $P_{1}=32+j$ & $\alpha_{1}=1.790^{\circ}$ & 2 & $0(+2)$ & $\infty$ & $\pm 3.580^{\circ}$ & $\pm 1.790^{\circ}$ & $\approx 1$ \\
\hline 5 & CORDIC & $P_{1}=64+j$ & $\alpha_{1}=0.895^{\circ}$ & 2 & $0(+2)$ & $\infty$ & $\pm 1.790^{\circ}$ & $\pm 0.895^{\circ}$ & $\approx 1$ \\
\hline 6 & Nano-rotations & $\begin{array}{l}P_{k}=512+j k \\
k=0, \ldots, 8\end{array}$ & $\alpha_{k}=k \cdot 0.112^{\circ}$ & 4 & $8(+2)$ & 15.50 & $\pm 0.895^{\circ}$ & $\pm 0.056^{\circ}$ & $\approx 1$ \\
\hline 6 bis & $\overline{\text { CORDIC }}$ & $\overline{P P_{1}=128+j}$ & $\alpha_{1}=0.448^{\circ}$ & $\overline{2}$ & $\overline{0(+2)}$ & $\infty$ & $\pm 0.895^{\circ}$ & $\pm 0.448^{\circ}$ & $\approx 1$ \\
\hline 7 bis & Nano-rotations & $\begin{array}{l}P_{k}=1024+j k \\
k=0, \ldots, 8\end{array}$ & $\alpha_{k}=k \cdot 0.056^{\circ}$ & 4 & $8(+2)$ & 17.50 & $\pm 0.448^{\circ}$ & $\pm 0.028^{\circ}$ & $\approx 1$ \\
\hline
\end{tabular}

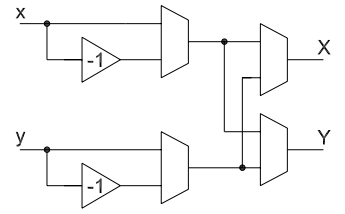

Fig. 8. Architecture of the trivial rotation (Stage 1).

It uses two negators, which are approximately equivalent to half an adder each, and four 2:1 multiplexers.

Stage 2: The second stage of the CORDIC II algorithm uses friend angles. It consists of the kernel $[25,24+j 7,20+j 15]$. The scale factor for all the coefficients is $R=25$, as $625=25^{2}=24^{2}+7^{2}=20^{2}+15^{2}$. Thus, there is no rotation error and $W L_{E}=\infty$. The friend angles that correspond to the coefficients are $0^{\circ}, 16.260^{\circ}, 36.870^{\circ}$, with normalized scaling $R_{\text {norm }}=1.563$, according to

$$
R_{\text {norm }}=\frac{R}{2^{\left\lfloor\log _{2} R\right\rfloor}} .
$$

The hardware architecture for the friend angle stage is shown in Fig. 9. It consists of five adders and seven 2:1 multiplexers, and can calculate all the rotations of the kernel depending on the configuration of the multiplexers. In Table II, two additional (+2) multiplexers are needed between stages in order to rotate the entire kernel (positive and negative rotations), as in [11].

Stage 3: The third stage of the CORDIC II algorithm uses the USR CORDIC. It consists of the kernel $[129,128+j 16]$, already shown in Table I. This stage reduces the remaining angle to $\pm 3.563^{\circ}$. The hardware architecture for the USR CORDIC stage is as shown in Fig. 3(b) for $k=4$. It consist

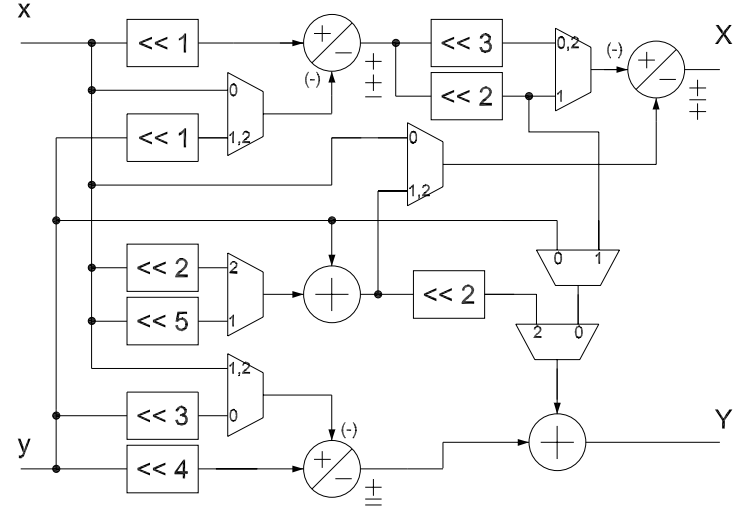

Fig. 9. Architecture of the friend angles (Stage 2).

of two adders and two 2:1 multiplexers.

Stages 4 and 5: The forth and fifth stages of the CORDIC II use conventional CORDIC rotations by $1.790^{\circ}$ and $0.895^{\circ}$.

Stage 6: The sixth stage uses nano-rotations. The kernel used is $P_{k}=512+j k, k=0, \ldots, 8$. The rotation angles of the kernel are $\alpha_{k}=k \cdot 0.112^{\circ}$. The remaining angle of the CORDIC II is $\pm 0.056^{\circ}$. The hardware circuit for the nanorotation stage is shown in Fig. 10. Figure 10(a) shows the nano rotator and Fig. 10(b) shows how the multiplication by $k$ is implemented. The decoder in Fig. 10(b) consists of a few logic gates.

Stages 6 bis and 7 bis: An alternative to the sixth stage of the CORDIC II is to add one more CORDIC rotation (stage 6 bis) followed by a nano-rotator (stage 7 bis), as shown in Table II. This increases the $W L_{E}$ of the nano-rotator and reduces the remaining angle of the CORDIC II bis to $\pm 0.028^{\circ}$. 


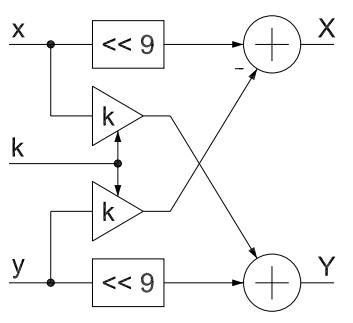

(a)

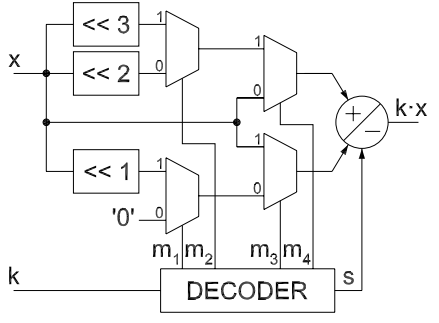

(b)
Fig. 10. Architecture of the nano-rotator (Stage 6). (a) Nano-rotator for the angle set $P_{k}=512+j k, k=0, \ldots, 8$. (b) Multiplication by $k$ for the nano-rotator, where $k \in\{0, \ldots, 8\}$.

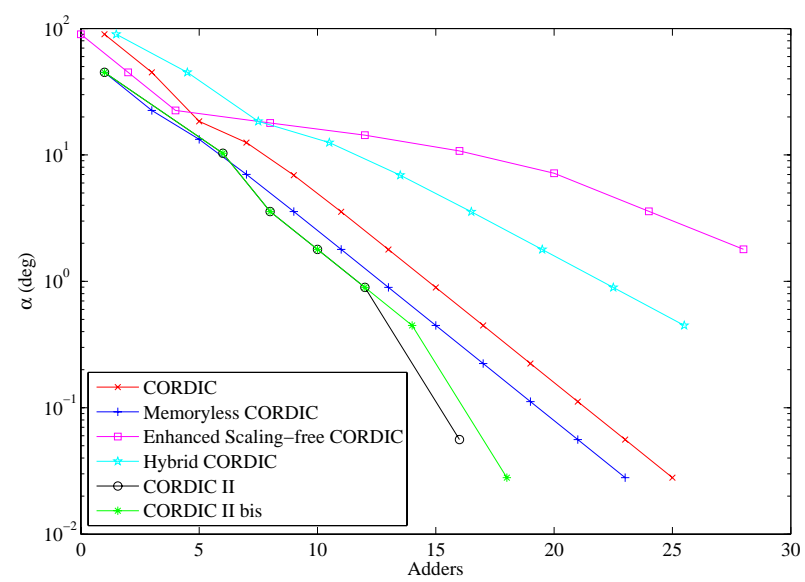

Fig. 11. Resolution of the rotators as a function of the number of adders.

Note also that the CORDIC II provides convergence for the entire circumference, as $\alpha_{\text {in }}$ for each stage is larger than $\alpha_{\text {out }}$ of the previous stage.

Finally, the control logic is similar to [11]: By representing the angle in the range $[0,1]$ the first three bits determine the trivial rotations, stages 2 and 3 use comparators, and the control for the rest of stages is obtained directly by representing the angle proportionally to the minimum rotation angle.

\section{COMPARISON}

Figure 11 compares the number of adders as a function of the remaining angle for the CORDIC, memoryless CORDIC [11], enhanced scaling-free CORDIC [7], hybrid CORDIC [4], CORDIC II and CORDIC II bis. For a precision of $\pm 0.056^{\circ}$, the CORDIC II uses 16 adders. This represents a saving of $30.4 \%$ with respect to the CORDIC algorithm and $23.8 \%$ with respect to the memoryless CORDIC. For a precision of $\pm 0.028^{\circ}$ the savings of the CORDIC II bis with respect to the CORDIC are $28 \%$.

Figure 12 shows the latency in terms of rotation stages. The proposed approaches reduce the latency of the CORDIC close to $50 \%$, and are only beaten by the hybrid CORDIC [4] at the cost of larger number of adders, as shown in Fig. 11.

Finally, we have obtained synthesis results for $65 \mathrm{~nm}$ ASIC technology. For a word length of 16 bits and aiming for $T_{c l k}=$ $4 \mathrm{~ns}$, the CORDIC II occupies $7816 \mu \mathrm{m}^{2}$ at $261 \mathrm{MHz}$. For the same constraints the CORDIC algorithm occupies $8599 \mu \mathrm{m}^{2}$ at $259 \mathrm{MHz}$. This represents savings of $10 \%$ in area of the CORDIC II with respect to the conventional CORDIC.

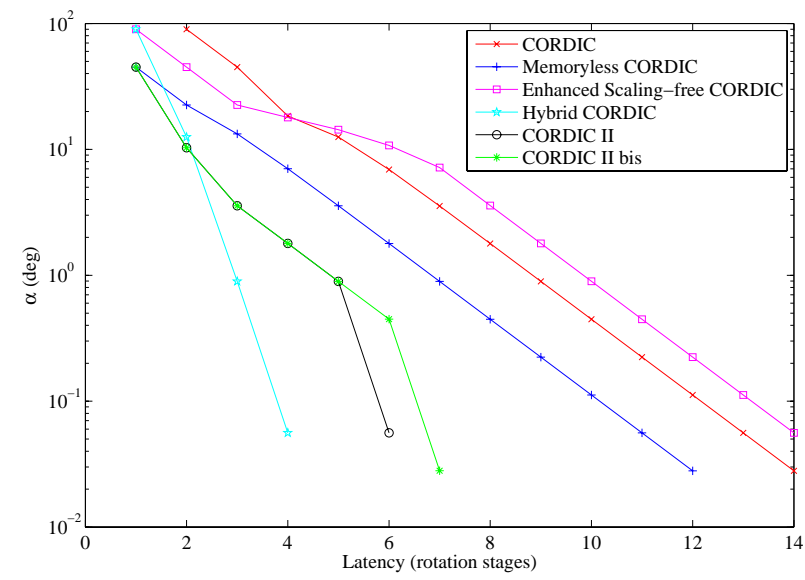

Fig. 12. Resolution of the rotators versus latency.

\section{CONCLUSIONS}

The CORDIC II is a new algorithm that substitutes the CORDIC micro-rotation by a new angle set. This involves three new types of rotators: friend angles, USR CORDIC and nano-rotations. By using the proposed micro-rotations, the CORDIC II requires the minimum number of adders among CORDIC algorithms so far.

\section{REFERENCES}

[1] J. E. Volder, "The CORDIC trigonometric computing technique," IRE Trans. Electronic Computing, vol. EC-8, pp. 330-334, Sep. 1959.

[2] M. Garrido, F. Qureshi, and O. Gustafsson, "Low-complexity multiplierless constant rotators based on combined coefficient selection and shift-and-add implementation (CCSSI)," IEEE Trans. Circuits Syst. I, vol. 61, no. 7, pp. 2002-2012, Jul. 2014.

[3] C.-S. Wu, A.-Y. Wu, and C.-H. Lin, "A high-performance/low-latency vector rotational CORDIC architecture based on extended elementary angle set and trellis-based searching schemes," IEEE Trans. Circuits Syst. II, vol. 50, no. 9, pp. 589-601, Sep. 2003.

[4] R. Shukla and K. Ray, "Low latency hybrid CORDIC algorithm," IEEE Trans. Comput., vol. 63, no. 12, pp. 3066-3078, Dec 2014.

[5] C.-S. Wu and A.-Y. Wu, "Modified vector rotational CORDIC (MVRCORDIC) algorithm and architecture," IEEE Trans. Circuits Syst. II, vol. 48, no. 6, pp. 548-561, Jun. 2001.

[6] S. Aggarwal, P. K. Meher, and K. Khare, "Area-time efficient scalingfree CORDIC using generalized micro-rotation selection," IEEE Trans. VLSI Syst., vol. 20, no. 8, pp. 1542-1546, Aug. 2012.

[7] F. Jaime, M. Sánchez, J. Hormigo, J. Villalba, and E. Zapata, "Enhanced scaling-free CORDIC," IEEE Trans. Circuits Syst. I, vol. 57, no. 7, pp. 1654-1662, July 2010.

[8] Y. Liu, L. Fan, and T. Ma, "A modified CORDIC FPGA implementation for wave generation," Circuits Syst. Signal Process., vol. 33, no. 1, pp. 321-329, 2014.

[9] C.-Y. Yu, S.-G. Chen, and J.-C. Chih, "Efficient CORDIC designs for multi-mode OFDM FFT," in Proc. IEEE Int. Conf. Acoust. Speech Signal Process., vol. 3, May 2006, pp. 1036-1039.

[10] C.-Y. Chen and C.-Y. Lin, "High-resolution architecture for CORDIC algorithm realization," in Proc. Int. Conf. Comm. Circuits Syst., vol. 1, Jun. 2006, pp. 579-582.

[11] M. Garrido and J. Grajal, "Efficient memoryless CORDIC for FFT computation," in Proc. IEEE Int. Conf. Acoust. Speech Signal Process., vol. 2, Apr. 2007, pp. 113-116.

[12] R. Andraka, "A survey of CORDIC algorithms for FPGA based computers," in Proc. ACM/SIGDA Int. Symp. FPGAs, Feb. 1998, pp. 191-200.

[13] P. K. Meher, J. Valls, T.-B. Juang, K. Sridharan, and K. Maharatna, "50 years of CORDIC: Algorithms, architectures, and applications," IEEE Trans. Circuits Syst. I, vol. 56, no. 9, pp. 1893-1907, Sep. 2009.

[14] M. Garrido, O. Gustafsson, and J. Grajal, "Accurate rotations based on coefficient scaling," IEEE Trans. Circuits Syst. II, vol. 58, no. 10, pp. 662-666, Oct. 2011.

[15] N. Takagi, T. Asada, and S. Yajima, "Redundant CORDIC methods with a constant scale factor for sine and cosine computation," IEEE Trans. Comput., vol. 40, no. 9, pp. 989-995, Sep. 1991. 